\title{
Construction of a high-density, high-quality genetic map of cultivated lotus (Nelumbo nucifera) using next-generation sequencing
}

Zhengwei Liu', Honglian Zhu', Yuping Liu', Jing Kuang ${ }^{1}$, Kai Zhou' ${ }^{1}$ Fan Liang ${ }^{2}$, Zhenhua Liư ${ }^{2}$ Depeng Wang ${ }^{2 *}$ and Weidong $\mathrm{Ke}^{1 *}$

\begin{abstract}
Background: The sacred lotus (Nelumbo nucifera) is widely cultivated in China for its edible rhizomes and seeds. Traditional plant breeding methods have been used to breed cultivars with increased yields and quality of rhizomes and seeds with limited success. Currently, the available genetic maps and molecular markers in lotus are too limited to be useful for molecular genetics based breeding programs. However, the development of next-generation sequencing (NGS) technologies has enabled large-scale identification of single-nucleotide polymorphisms (SNPs) for genetic map construction. In this study, we constructed an SNP-based high-density genetic map for cultivated lotus using double digest restriction site-associated DNA sequencing (ddRADseq).

Results: An $F_{2}$ population of 96 individuals was derived from a cross between the rhizome lotus cultivar 'Juwuba' (male parent) and the seed lotus cultivar 'Mantianxing' (female parent). Genomic DNAs from this population were digested with the restriction enzymes EcoRl and Mspl and then sequenced. In total, $133.65 \mathrm{~Gb}$ of raw data containing 1,088,935,610 pair-end reads were obtained. The coverage of reads on a reference genome was $7.2 \%$ for the female parent, $6.56 \%$ for the male parent, and $1.46 \%$ for $F_{2}$ individuals. From these reads, 10,753 valid SNP markers were used for genetic map construction. Finally, 791 bin markers (so-segregated adjacent SNPs treated as a bin marker), consisting of 8,971 SNP markers, were sorted into 8 linkage groups (LGs) that spanned $581.3 \mathrm{cM}$, with an average marker interval of $0.74 \mathrm{cM}$. A total of 809 genome sequence scaffolds, covering about $565.9 \mathrm{cM}$ of the wild sacred lotus genome, were anchored on the genetic map, accounting for $70.6 \%$ of the genome assembly.
\end{abstract}

Conclusions: This study reports the large-scale discovery of SNPs between cultivars of rhizome and seed lotus using a ddRADseq library combined with NGS. These SNPs have been used to construct the first high-density genetic map for cultivated lotus that can serve as a genomic reference and will facilitate genetic mapping of important traits in the parental cultivars.

Keywords: Double digest RADSeq, Nelumbo nucifera, Molecular breeding, Assembly anchoring, Single-nucleotide polymorphisms, Next generation sequencing, Genetic map

\section{Background}

Nelumbo nucifera Gaertn., also known as sacred lotus, is an aquatic crop of considerable agricultural, ornamental, religious, and medical importance $[1,2]$. Sacred lotus belongs to the family Nelumbonaceae $(2 \mathrm{n}=16$, genome size is about $929 \mathrm{Mb}$ ), which comprises only two species,

\footnotetext{
*Correspondence: wangdp@nextomisc.org; wdke63@163.com

${ }^{2}$ Nextomics Biosciences Co., Ltd., Wuhan, Hubei, China

'Institute of Vegetable, Wuhan Academy of Agriculture Science and

Technology, Wuhan, Hubei 430065, China
}

N.nucifera and N.lutea [3]. Analyses of morphological differences and molecular markers have identified three distinct types of cultivar: rhizome lotus, seed lotus, and flower lotus $[4,5]$. Rhizome lotus and seed lotus are the two most popular aquatic vegetable crops in China [6] with estimated areas under cultivation of 660,000 and 67,000 ha, respectively, in 2012.

Lotus breeding began about 30 years ago in China [7] and several elite lotus cultivars have been produced and are now widely cultivated [8]. Nevertheless, research on 
lotus still faces many difficulties compared to other crop species as it is labor-intensive, time consuming, and costly. Firstly, single lotus plants can span three to five square meters and need to be grown in separate cement ponds to prevent misidentification of individuals, which means a high investment in the cultivation facility. Secondly, the underground rhizomes of lotus can expand to 30 to $50 \mathrm{~cm}$ in depth in the soil, which increases the difficulty of rhizome phenotyping. As a result of these complications, the genetic basis of the most important agronomic traits and other phenotypes are not yet understood.

Plant breeding using molecular genetic markers is an efficient approach to overcoming such limitations. Highdensity genetic maps, including quantitative trait locus (QTL) mapping and marker-assisted selection, are essential for the efficient use of this approach to plant breeding. Yang et al. [9] reported the first genetic maps for $N$. nucifera and N. lutea based on 47 and 177 SSR (simple sequence repeat) markers, respectively. These maps were later expanded by Zhang et al. [1, 10]. Currently, the N.nucifera genetic map comprises 224 markers and the $N$. lutea genetic map has 3895 RADseq markers and 156 SSR markers on 9 linkage groups (LGs). These genetic maps are of value for mapping quantitative trait loci (QTLs) affecting plant size, leaf shape, petal shape and color, and other desirable characters; however, they are less useful for mapping yield-related traits in rhizome and seed lotuses since the parental plants were wild lotus phenotypes that exhibit unfavorable agronomic traits such as thin rhizomes and low seed yield. In addition, the existing genetic map for $N$. nucifera also lacks sufficient markers to conduct QTL analysis and molecular mapping, while the high-density genetic map for $N$. lutea has failed to coalesce into eight linkage groups representing the eight lotus chromosomes. As a result, construction of a more saturated map with a higher density of markers is needed to meet the demand for breeding improved lotus cultivars especially for crop varieties.

Next-generation sequencing (NGS) provides the opportunity for large-scale genome sequencing, and has already been exploited in denovo sequencing of disparate organisms such as panda [11], cucumber [12], apple [13], and lotus [1, 2]. Ming et al. successfully sequenced the genome of the sacred lotus cultivar 'China Antique' [1], while a draft genome of a wild strain of lotus was reported that spanned $792 \mathrm{Mb}, 85.2 \%$ of the estimated $929 \mathrm{Mb}$ lotus genome. Single-nucleotide polymorphisms (SNPs) are the most abundant and stable form of genetic variation in most genomes. Large-scale identification of high quality SNPs can be achieved through use of restriction site-associated DNA sequencing (RADseq); this method has the advantages of relatively low cost and speed [14], which are highly beneficial for genetic map construction. RADseq is based on sequencing the DNA flanking specific restriction enzyme sites rather than the whole genome [15]. The use of two restriction enzymes, double digest RADseq (ddRADseq), improves the efficiency of producing a sequencing library and robustness of the results while minimizing cost [16].

Rhizome and seed lotus cultivars display high phenotypic diversity in both vegetative growth and sexual reproduction (Fig. 1). In addition, high genetic diversity has been found between the two types of cultivar $[4,17]$. Consequently, a map derived from a cross between a rhizome lotus cultivar and a seed lotus cultivar will substantially facilitate the molecular characterization of phenotypic variation and QTL mapping of important trait loci in both types of lotus cultivar.

In this study, a high-density genetic map was constructed using ddRADseq [16] of genomic DNA from $96 F_{2}$ progeny derived from a cross between the rhizome lotus cultivar $N$. nucifera cv. 'Juwuba' and the seed lotus cultivar N. nucifera cv. 'Mantianxing'. Overall, $71.3 \%$ of the assembled genome of the wild Asian lotus [2] was anchored. This is the first report of a genetic map based on two types of lotus cultivar and will be of value for accelerating the characterization of agronomically important traits.

\section{Results}

Phenotypic differences between parental cultivars

Although the two cultivars used here, 'Juwuba' and 'Mantianxing', belong to the same species, they exhibit distinct phenotypic differences. 'Juwuba' has vigorous vegetative growth, an enlarged rhizome, and a small number of white flowers; by contrast, 'Mantianxing' exhibits vigorous sexual reproduction with a long flowering time and large numbers of red flowers with extra carpels, but its rhizome is smaller (Fig. 1). We have recorded the main characters of both parents for five years (data not show). The rhizome weight was 7.1 fold greater in 'Juwuba' than 'Mantianxing' and the width of the section between the third and fourth knots of the main stem was 1.54 fold greater in 'Juwuba'. The numbers of flowers and carpels in 'Mantianxing' were 8 and 1.34 fold higher, respectively, than 'Juwuba'. From the wide phenotypic differences in the parents, we anticipated that broad variation would be present in the $F_{2}$ generation.

\section{Restriction enzyme selection for DNA sequencing library construction}

The assembly sequence of the wild lotus [2] was in silico digested with five enzyme combinations EcoRI/SbfI, EcoRI/MspI, EcoRI/SphI, SphI/MluCI, and NlaIII/MluCI. The distribution of restriction fragments on the genome was predicted. As shown in Additional file 1, the MspI/ 


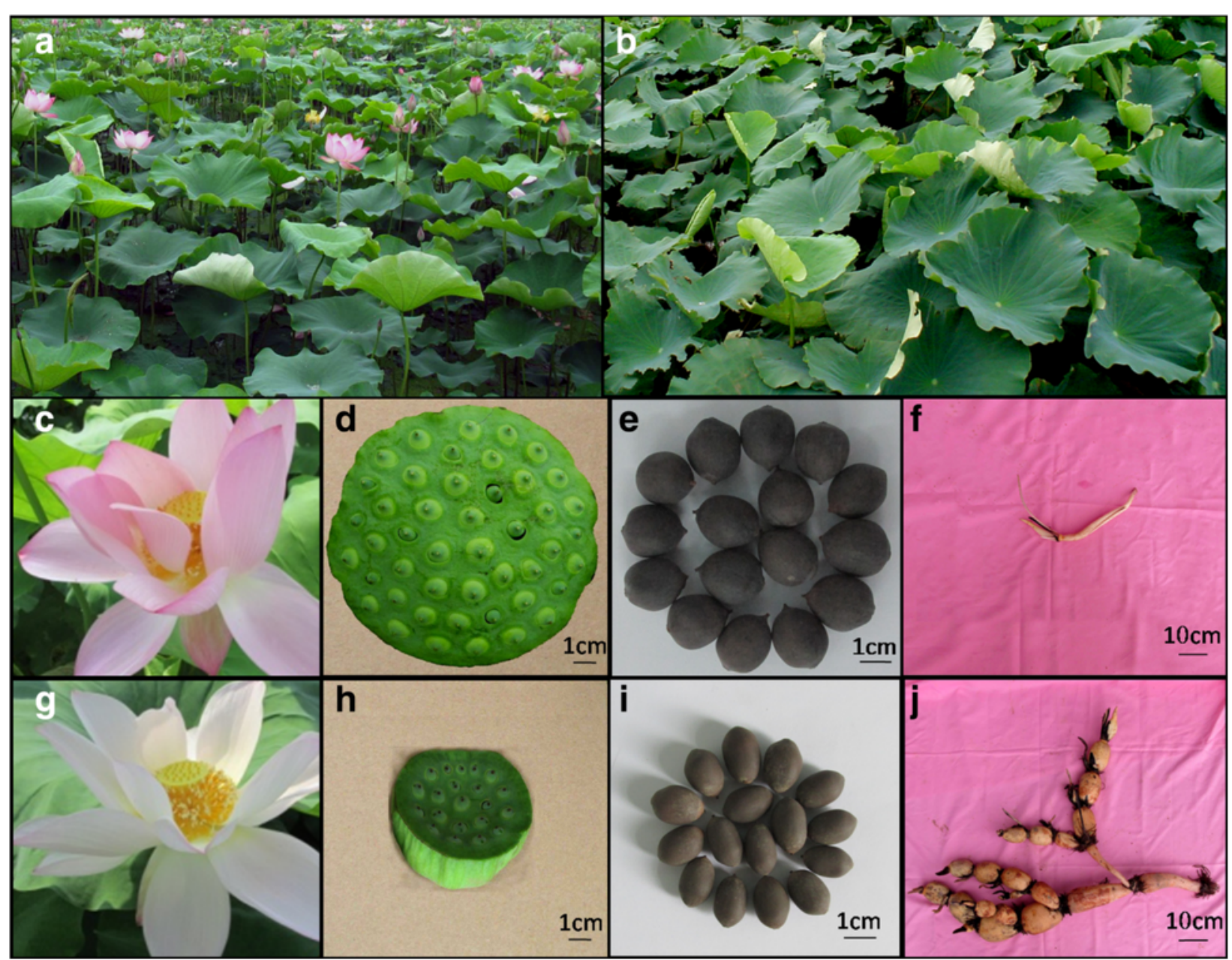

Fig. 1 The main phenotypic differences between parent cultivars. a the seed lotus cultivar 'Mantianxing' used as female parent was planted in field in fast-growing stage; $\mathbf{b}$ the rhizome cultivar 'Juwuba' used as male parent was planted in field in fast-growing stage; $\mathbf{c}$, $\mathbf{d}$, e and $\mathbf{f}$ is the flower, fruit, seed and rhizome of 'Mantianxing', respectively; $\mathbf{g}, \mathbf{h}, \mathbf{i}$ and $\mathbf{j}$ is the flower, fruit, seed and rhizome of Juwuba, respectively

EcoRI combination was predicted to produce 157,936 restriction fragments of 250 to $500 \mathrm{bp}$; this was the maximum predicted fragment number of the five enzyme combinations, and was considered sufficient to construct a high-density genetic map. Therefore, we double digested the genomic DNA of parents and progeny with MspI and EcoRI to construct a sequencing library.

\section{DNA sequencing}

The ddRADseq library of all samples was analyzed by massively parallel Solexa sequencing, which generated a total of $\sim 133.65 \mathrm{~Gb}$ raw data containing 1,088,935,610 pair-end reads of $\sim 100 \mathrm{bp}$ (Table 1). After removing the low-quality reads ( $>5$ bases with Q score $>20$ ), approximately $89.44 \mathrm{~Gb}$ clean reads were sorted based on identified barcodes. Most of the samples had more than 5 million reads (Fig. 2a, Additional file 2). Sample z35, which only had 1.2 million reads, was omitted from the following analysis.

The sorted reads were aligned to the wild strain lotus genome assembly (792 $\mathrm{Mb})$ [2] using the BurrowsWheeler Aligner (BWA) program (v0.7.10) [18]. The $17,741,926$ mapped reads from the male parent, Juwuba, and the 14,359,968 reads from the female parent, Mantianxing, covered 7.2 and $6.56 \%$ of the lotus genome, respectively. For $\mathrm{F}_{2}$ individuals, an average of $7,377,795$ reads was mapped to the reference genome [2] with mean coverage of $1.46 \%$ of the lotus genome. The read depth was $57.94 \times$ for the female parent, $78.57 \times$ for the male parent, and $48.35 \times$ for the $F_{2}$ progeny (Fig. $2 b$ ). Detailed read information for the parents and the $F_{2}$ individuals is shown in Additional file 2. The raw data of the $F_{2}$ individuals contained many highly redundant reads, which may have resulted from chloroplasts in the leaf tissues that were used for DNA extraction. This 
Table 1 Summary of RAD sequencing for SNP calling from the rhizome lotus Juwuba and the seed lotus Mantianxing (N. nucifera) and their F2 progenies

\begin{tabular}{|c|c|c|c|c|c|c|c|}
\hline Sample & Total reads & Total base (Gb) & Mapped reads & Mapped base (Gb) & Alignment rate (\%) & Coverage (\%) & Depth $(X)$ \\
\hline Mantianxing & $16,429,464$ & 1.64 & $14,359,968$ & 1.44 & 87.40 & 7.20 & 57.94 \\
\hline Juwuba & $19,032,200$ & 1.90 & $17,741,926$ & 1.77 & 93.22 & 6.56 & 78.57 \\
\hline Average of $F_{2}$ population & $11,136,905$ & 1.38 & $7,377,795$ & 0.91 & 65.76 & 1.46 & 48.35 \\
\hline Total & $1,088,935,610$ & 133.65 & $730,348,710$ & 89.44 & 65.00 & 1.70 & 48.65 \\
\hline
\end{tabular}

Note: total line, all samples were calculated including parents and $F_{2}$ population

contaminating DNA reduced the mapping rate for $F_{2}$ individuals.

\section{SNP calling and genotyping}

The mapped reads of parents and $F_{2}$ individuals were then aligned to the reference genome [2] for SNP calling. Only uniquely mapped reads with one or two mismatches were retained. SNP calling was carried out using the Genome Analysis Toolkit program (v3.1-1) [19] and filtered using a series of stringent selection criteria (detailed in the Methods section). At last, 10,753 valid SNP markers were obtained and the genotyping loci ranged from 8,243 to 10,747 with high integrity. The SNP markers integrity was 99.38 \% for 'Mantianxing', $98.07 \%$ for 'Juwuba', and $97.36 \%$ for the $F_{2}$ population (Fig. 2c).

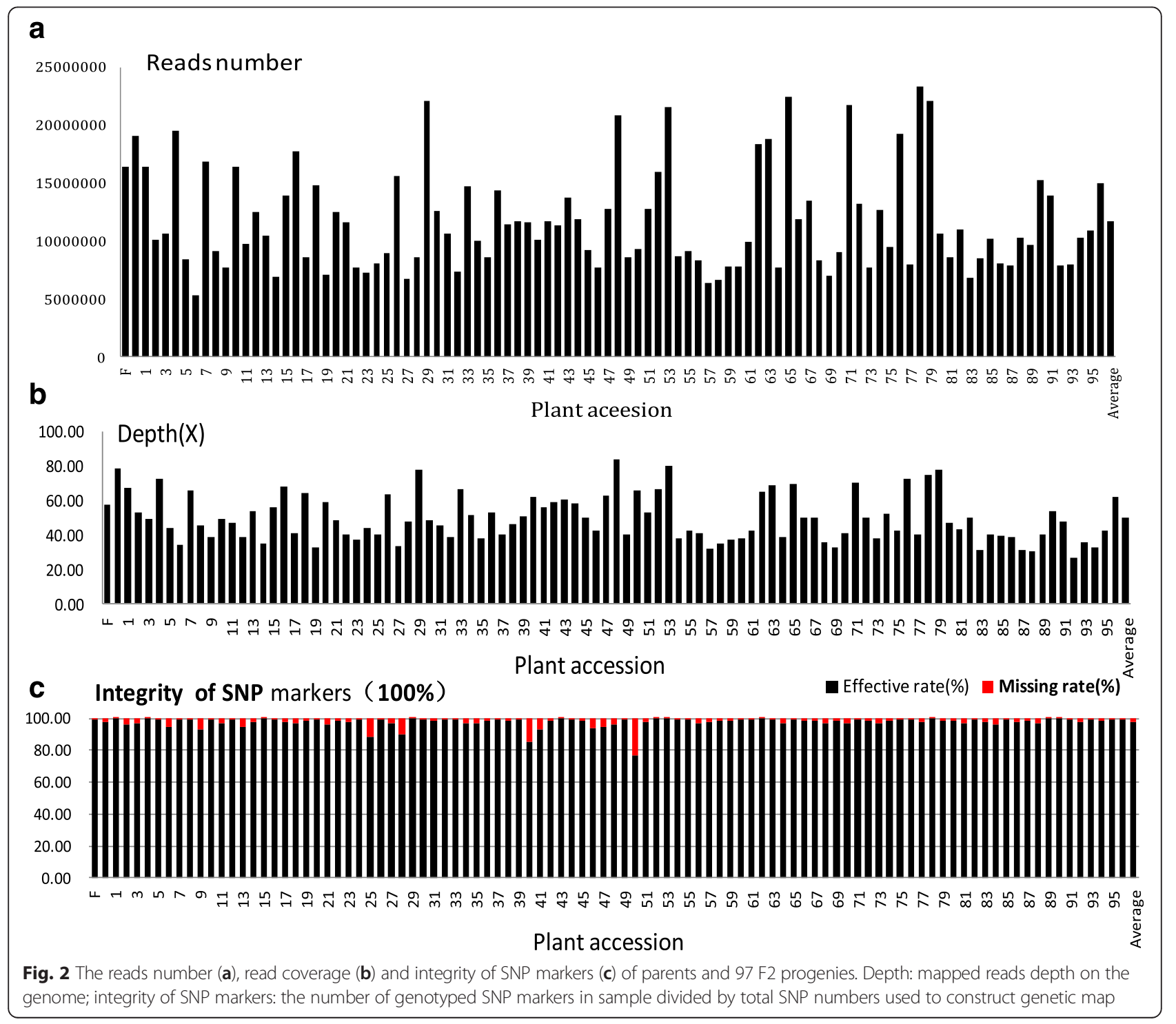


Table 2 Summary of eight linkage groups of high-density genetic map

\begin{tabular}{|c|c|c|c|c|c|c|c|}
\hline Linkage group & SNP markers & Bin markers & Linkage distance (CM) & Mean distance (cM) & Largest gap (cM) & $\begin{array}{l}\text { No. of distorted segregation } \\
\text { markers }(P<0.05)\end{array}$ & No. of SDRs \\
\hline$\overline{L G 1}$ & 3,131 & 253 & 188.7 & 0.75 & 4.0 & 22 & 1 \\
\hline LG2 & 1,416 & 100 & 78.4 & 0.78 & 3.9 & 9 & 2 \\
\hline LG3 & 1,155 & 104 & 40.2 & 0.39 & 4.5 & 23 & 4 \\
\hline LG4 & 682 & 73 & 48.5 & 0.66 & 2.7 & 10 & 2 \\
\hline LG5 & 716 & 79 & 64.5 & 0.82 & 4.3 & 4 & 1 \\
\hline LG6 & 760 & 70 & 63.3 & 0.90 & 8.4 & 7 & 1 \\
\hline LG7 & 719 & 56 & 48.9 & 0.87 & 3.3 & 10 & 2 \\
\hline LG8 & 392 & 56 & 49.0 & 0.87 & 2.90 & 3 & 0 \\
\hline Total & 8,971 & 791 & 581.4 & 0.74 & - & 88 & 13 \\
\hline
\end{tabular}

Note: SDRs, segregation distorted regions

\section{Genetic map construction}

The 10,753 SNP markers segregating in the $F_{2}$ population were used to construct a genetic linkage map using JoinMap4.1 software [20]. At a LOD threshold of 15, a total of 8,971 SNP markers were clustered into 8 LGs, consistent with the haploid chromosome number of lotus $(n=8)$. The types of the grouped SNP markers in the parents and the $F_{2}$ population are shown in Additional file 3. The SNP markers were next scanned in $100 \mathrm{~kb}$ windows on the mapped scaffolds of the reference genome, and co-segregating SNP markers were sorted into bin markers.

In total, 791 bin markers were identified after scanning for co-segregation and were used to construct a genetic linkage map with 8 LGs spanning $581.4 \mathrm{cM}$ and an average marker interval of $0.74 \mathrm{cM}$ (Table 2, Additional file 4). Detailed information on the SNPs and the bin markers on the linkage map are shown in Additional file 5. LG sizes varied widely: the largest group, LG1, contained 253 bin markers spanning 188.7 cM, while the shortest, LG8, only included 100 markers over $40.2 \mathrm{cM}$. The average marker interval of the eight LGs ranged from 0.39 (LG4) to $0.90 \mathrm{cM}$ (LG6) (Table 2).

\section{Segregation-distorted markers}

Distorted segregation $(P<0.05)$ was found for 88 of the mapped bin markers, i.e., $11.1 \%$ of the total (Table 2). The majority of the markers showing segregation distortion were distributed as clusters; in line with previous studies, we defined clusters of more than three adjacent loci showing significant segregation distortion as segregation distortion regions (SDRs) [10, 11]. Thirteen SDRs distributed across seven linkage groups were identified (all LGs except LG8), with the largest number (4 SDRs) on LG3 (Fig. 3; Table 2). These segregation distortion markers may result from gametic or zygotic selection [12], but their presence does not have a large effect on further use of the map for QTL mapping [13-15]. Therefore, the segregation distortion markers were retained here to increase the coverage of the linkage groups.

\section{Genome scaffolds anchoring of the wild strain of sacred lotus}

In our previous work, we partially sequenced the genome of the wild Asian lotus and generated a construct of $792 \mathrm{Mb}$ that accounted for 85 to $95 \%$ of the estimated complete lotus genome [1]. Here, we anchored 809 scaffolds to eight lotus pseudochromosomes with a total length of $565 \mathrm{Mb}$, representing $71.3 \%$ of the lotus assembly (Table 3); the positions of the scaffolds on the eight pseudochromosomes are detailed in Additional file 5. Of these anchored scaffolds, 164 (20.3 \%) could be oriented. The number of anchored scaffolds ranged from

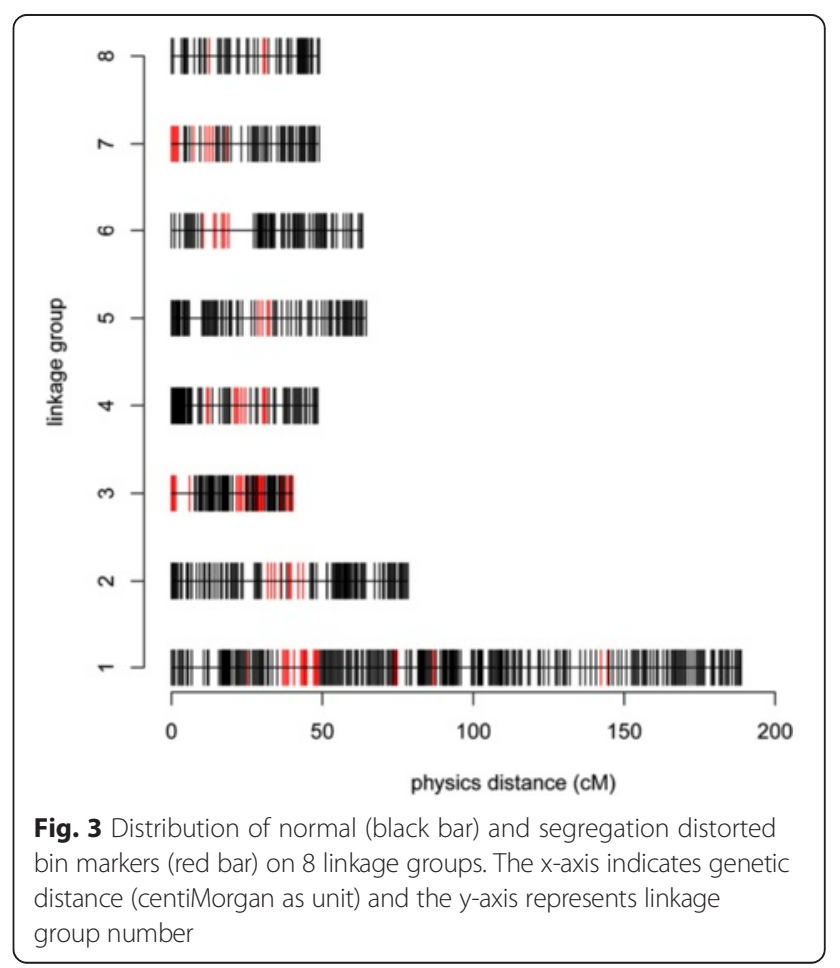


Table 3 The scaffold number, total length, physical size, marker density and effective length of eight pseudochromosomes

\begin{tabular}{|c|c|c|c|c|c|}
\hline Pseudochromosome & No. of Scaffold & Total length (bp) & Linkage distance (cM) & $\mathrm{Mb} / \mathrm{cM}$ & Effective length (bp) \\
\hline chr1 & 236 & $177,951,281$ & 188.7 & 1.06 & $173,848,305$ \\
\hline chr2 & 149 & $90,702,750$ & 78.4 & 0.86 & $88,660,738$ \\
\hline chr3 & 129 & $77,900,568$ & 40.2 & 0.52 & $76,335,923$ \\
\hline chr4 & 67 & $48,236,206$ & 48.5 & 1.01 & $47,320,720$ \\
\hline chr5 & 74 & $52,360,038$ & 64.5 & 1.23 & $51,365,207$ \\
\hline chr6 & 77 & $49,297,711$ & 63.3 & 1.28 & $48,249,537$ \\
\hline chr7 & 50 & $48,522,756$ & 48.9 & 1.01 & $47,495,387$ \\
\hline chr8 & 27 & $20,903,902$ & 49.0 & 2.34 & $20,529,685$ \\
\hline Total & 809 & $565,875,212$ & 581.4 & 1.03 & $553,805,502$ \\
\hline
\end{tabular}

Note: total length, pseudochromosome length containing unknown base pairs in genome assembly; effective length, the pseudochromosome length without unknown base pairs

27 for LG8 to 236 for LG1; the physical sizes of the eight pseudochromosomes ranged from $177.95 \mathrm{Mb}$ (LG1) to $20.90 \mathrm{Mb}$ (LG8). The relationship between genetic distance (in $\mathrm{cM}$ ) and physical size (in $\mathrm{Mb}$ ) varied widely, from $0.52 \mathrm{cM} / \mathrm{Mb}$ on LG3 to $2.34 \mathrm{cM} / \mathrm{Mb}$ on LG3, with an average of $1.03 \mathrm{cM} / \mathrm{Mb}$.

\section{Comparative genomics}

Next, we compared our newly generated genetic map with the two published SSR-based maps [9]. We analyzed 137 SSR markers from the $N$. lutea cultivar 'AL1' and 36 SSR markers from the N.nucifera cultivar 'China Antique'. Results showed that 111 and 30 markers respectively were shared with the wild lotus genome [9], and 58 and 19 markers, respectively, could be mapped to the anchored scaffolds of our new genetic map. The correspondence between genetic maps is shown in Table 4. Most, if not all, markers on LG1-M and LG7-M (LG-M = linkage group of male parent) from N.lutea were assigned to LG1 in our genetic map (Fig. 4). In agreement with Zhang et al., who integrated LG4-M and LG1-M into LG1 of the RADseq marker-based group [10], we infer from our results that LG1-M, LG4$\mathrm{M}$, and LG7-M all belong to LG1 in our map. The $N$. lutea markers on LG2-M predominantly fell into LG4, LG3-M into LG7, LG5-M into LG5, LG6-M into LG6, LG8-M into LG3, LG9-M into LG2, and LG10-M into LG8 of our genetic map.

We also compared our genetic map with a high density American lotus map [10]. We found that most SSR markers on LG5 from the high density American lotus map fell into LG1 in our map. Considering that the LG1s from the two genetic maps analyzed above belong to the same chromosome, we can further integrate LG1 and LG5 from the American lotus into one linkage group.

\section{Discussion}

Molecular genetic markers provide a powerful tool for associating heritable traits with underlying genetic variation. However, due to technical limitations, most of the initial large-scale genetic marker development and high density genetic map construction was carried out in model species, such as Arabidopsis [21] and rice [22]. In recent years, the development of NGS technology, especially ddRADseq, has provided a powerful and low-cost tool for the large-scale discovery of SNP markers in any plant species $[15,23]$. This approach has been successfully

Table 4 The correspondence between our genetic map and two published SSR-based maps [9] for scoring SSR markers

\begin{tabular}{|c|c|c|c|c|c|c|c|c|c|c|c|c|c|c|c|c|c|}
\hline \multirow{2}{*}{$\begin{array}{l}\text { Mantianxing } \times \\
\text { Juwuba }\end{array}$} & \multicolumn{7}{|c|}{ Male parent linkage group (number of SSR markers) } & \multicolumn{10}{|c|}{ Female parent linkage group (number of SSR markers) } \\
\hline & 1 & 2 & 3 & 4 & 5 & 6 & 7 & 1 & 2 & 3 & 4 & 5 & 6 & 7 & 8 & 9 & 10 \\
\hline LG1 & 0 & 4 & 1 & 0 & 3 & 0 & 2 & 12 & 1 & 2 & 2 & 0 & 0 & 6 & 0 & 0 & 0 \\
\hline LG2 & 0 & 0 & 0 & 0 & 0 & 0 & 0 & 0 & 0 & 0 & 0 & 0 & 0 & 0 & 0 & 2 & 0 \\
\hline LG3 & 1 & 0 & 1 & 0 & 0 & 0 & 0 & 1 & 0 & 0 & 0 & 0 & 0 & 1 & 5 & 0 & 0 \\
\hline LG4 & 0 & 0 & 1 & 0 & 0 & 0 & 0 & 0 & 8 & 0 & 0 & 0 & 1 & 0 & 0 & 0 & 0 \\
\hline LG5 & 0 & 0 & 0 & 0 & 0 & 0 & 0 & 0 & 0 & 0 & 0 & 1 & 0 & 0 & 0 & 0 & 0 \\
\hline LG6 & 2 & 0 & 0 & 1 & 0 & 0 & 0 & 0 & 0 & 0 & 0 & 0 & 5 & 0 & 0 & 0 & 0 \\
\hline LG7 & 2 & 0 & 0 & 0 & 0 & 0 & 0 & 0 & 1 & 6 & 1 & 0 & 1 & 0 & 0 & 0 & 0 \\
\hline LG8 & 0 & 0 & 0 & 0 & 0 & 1 & 0 & 0 & 0 & 0 & 0 & 0 & 0 & 0 & 0 & 0 & 1 \\
\hline
\end{tabular}

Note: numbers in the table are shared SSR markers between Mantianxing $\times$ Juwuba map and the lotus genetic map constructed by Yang et al. ( the male and female genetic map) [9] 


\section{LG7-M}

\section{LG1}

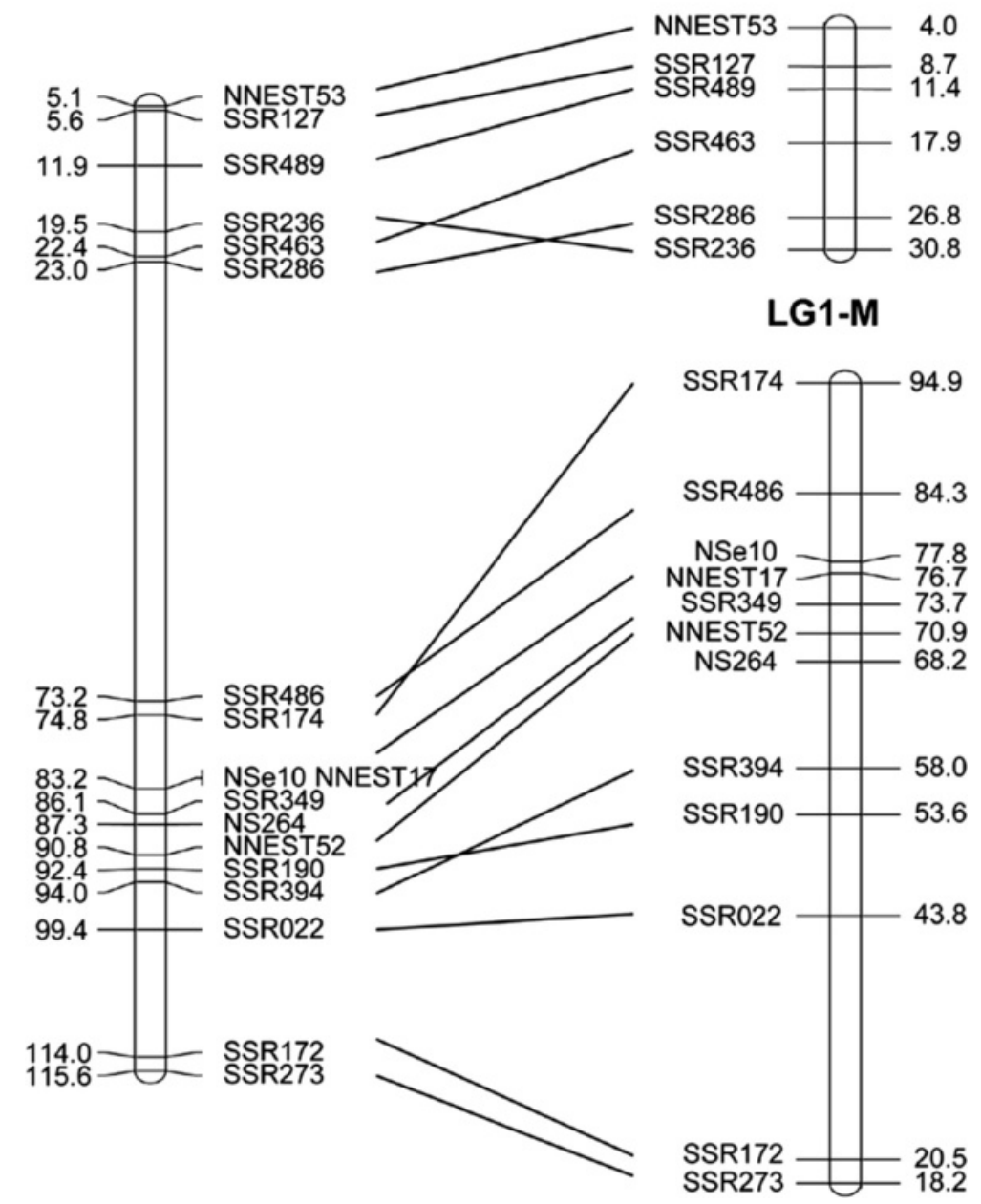

Fig. 4 Comparison of LG1 in this study with LG1-M and LG7-M in SSR-based maps [9]. SSR marker information came from Yang et al. [9]

applied to the construction of high-density genetic maps $[24,25]$, assembly of genome scaffolds [26-28], and mapping of QTLs [29, 30].

In the present study, we successfully constructed a high-density genetic map from two N.nucifera cultivars using the ddRADseq method. We identified 791 bin markers distributed across 8 LGs; the number of LGs exactly matched the number of chromosomes in Nelumbo species $(n=8)$. The average interval between genetic bin markers in this study is $0.74 \mathrm{cM}$. This is comparable with or better than the marker density of genetic maps constructed for other crops using the same RADseq protocol, e.g.,sesame,1,272 markers and 1.2 cM interval [31], soybean, 5,785 markers and $0.43 \mathrm{cM}$ interval [32], and peanut, 1,685 markers and $0.9 \mathrm{cM}$ interval [33]. In addition, our new genetic map provides a marked improvement in marker density and population size compared to the two previously published genetic maps for N.nucifera and N.lutea [10], which were based on the same $F_{1}$ population of 51 individuals. The published map for $N$. nucifera contains 224 markers, and that of $N$. lutea has 3,895 RADseq markers and 156 SSR markers on 9 LGs. The genetic map produced in the current study is therefore a more saturated map and has high resolution for QTL mapping.

Using the 8,971 ddRAD markers in the present high density genetic map, 809 genome scaffolds of the wild strain $N$. nucifera assembly (from uncultivated sacred lotus) were successfully anchored to eight pseudochromosomes with a total length of $566 \mathrm{Mb}(71.3 \%$ of the lotus genome). The previously published scaffold assembly for lotus consists of nine megascaffolds indicating that a gap was present within a linkage group of one chromosome. The additional anchoring in this study resulted in a match between the number of linkage groups and the haploid number of chromosomes; moreover, the 
relative sizes of the linkage groups are similar to those of the chromosomes [34]. Therefore, our new genetic map, along with the improved genome assembly, resolution, and integrity, will play an important role in further marker assisted selection and QTL mapping of important traits for breeding new varieties of lotus.

Segregation distorting is a common phenomenon in most genetically segregating populations [35-37] and is thought to be an important driving force in evolution through increasing genomic heterozygosity. We also observed a low rate of segregation distortion affecting some markers in our genetic map. The rate of such markers was less than one-third that reported for the SSR based American map [8]. Two factors may account for this difference. Firstly, our map used an $F_{2}$ population whereas the American map was based on an $F_{1}$ population. In general, segregation marker skewing is lower in $F_{2}$ populations than in recombinant inbred lines, backcross populations, and $F_{1}$ populations [38]. Secondly, the two Nelumbo species used here as parents show less genetic divergence than is present between the American lotus and the Asian lotus. Mapping based on wide crosses carries the risk of distorted segregation and altered linkages due to gametic selection and/or chromosomal rearrangements [35]. We further found that most of the aberrant segregation markers tended to be located in close proximity and 11 putative SDRs were detected; this indicated that segregation distortion was not induced by technical problems but most likely arose through biological factors such as selection for gametophytes and sporophytes.

Comparison of genetic maps for N.nucifera and $N$. lutea showed good collinearity in all the linkage groups (Fig. 4). Markers on LG1-M and LG7-M of N. lutea generally appeared in the same order as those from LG1 of N.nucifera, although a few adjacent markers showed a reversal in their order. The discrepancies in the order of adjacent markers might be due to the relatively small populations used for linkage map construction (51 individuals for the genetic map of $N$. lutea vs. 96 individuals for N.nucifera); however, we cannot rule out the possibility of genetic variation between the two species. The long genetic length and high density of markers in our genetic map provided the opportunity to fill gaps in previous maps of N.lutea. We successfully integrated LG1-M, LG4-M, and LG7-M into a single linkage group (LG1). Moreover, we integrated LG1 and LG5 from the RADseq map [10] into a single linkage group, which further saturated the high density map for American lotus.

Although $96 \mathrm{~F}_{2}$ progeny were used to construct the genetic map and 8972 SNP markers $(0.064 \mathrm{cM}$ per SNP marker) were obtained, only 791 bin markers were identified due to co-segregation in the $F_{2}$ population. The small genetic pool resulted in lower recombination in the $F_{2}$ individuals and reduced the resolution of the genetic map [27]. Currently, we are using asexual reproduction of $F_{1}$ progeny to increase the number of $F_{2}$ progeny to over 300 . Using this expanded resource, we will soon obtain more SNP markers to further saturate our genetic map. As the $F_{2}$ population can be preserved permanently through asexual reproduction, this will facilitate the characterization of important traits in the laboratory and field.

\section{Conclusions}

In this paper, we use cutting-edge genomic techniques to create genetic map of $N$. nucifera that greatly improves on the quality of previous genetic maps. $N$. nucifera is valued as a food crop in addition to its ornamental and cultural importance, yet no genetic map that we are aware of focuses on agricultural cultivars. We have filled that gap. In addition, because of the higher density and quality of information in this genetic map, we are able to anchor the "scaffold" portions of the recorded $N$. nucifera genome assembly that are not based on continuous, contiguous sequences. With a better genome assembly, the genotypic basis of valuable traits can be identified, and sacred lotus breeding can be practiced more efficiently.

\section{Methods}

\section{Plant breeding and DNA extraction}

Two cultivars of $N$. nucifera were used in this study: 'Juwuba', a high-rhizome-yield cultivar, and 'Mantianxing', a high-seed-yield cultivar. They are the most popular rhizome and seed lotus cultivars grown in the Yangtze valley. These two accessions were developed and preserved by National Germplasm Wuhan Aquatic Vegetable Garden, Institute of Vegetable, Wuhan Academy of Agriculture Science and Technology and can be open accessed (ATTEN: Weidong Ke, email: wdke63@163.com). Lotuses are not endangered or protected species in China and lotus research and field studies were authorized by local government. The parental generations and $\mathrm{F}_{2}$ plants were planted and preserved through asexual reproduction in six- $\mathrm{m}^{2}$ containers individually at the National Germplasm Wuhan Aquatic Vegetable Garden in Wuhan city. Key morphological and agronomical characteristics of both parents were analyzed.

For DNA extraction, young leaves of the parental plants were harvested from the underground stem tip developed from the rhizome; for $F_{2}$ individuals, the primary leaves from germinating seeds were used as young leaves on underground stem tips were too short. Approximately $0.3 \mathrm{~g}$ young leaves per sample were collected, treated with liquid nitrogen and grounded into powder. Genomic DNA was then extracted using the cetyltrimethyl ammonium bromide (CTAB) method [39] 
with the minor modification that $4 \%$ PVP 40 was added to the CTAB buffer.

\section{In silico analysis of restriction enzyme-recognition sites on the reference lotus genome}

The sequence of the wild strain lotus genome assembly [2] was used to identify the best restriction enzyme combination for sequencing library construction. The assembly is $792 \mathrm{Mb}$ in length and includes 3031 scaffolds ( $>2 \mathrm{~kb}$ ); scaffold N50 was $986.5 \mathrm{~kb}$ (a long scaffold N50 generally indicates a high-quality assembly). In silico double-restriction digestion of the lotus genome with five enzyme combinations was carried out using Perl script developed by ourselves (https:/github.com/ Nextomics/ddRAD-pipeline). The tested enzyme combinations were EcoRI/SbfI, EcoRI/SphI, SphI/MluCI, EcoRI/MspI and NlaIII/MluCI. This in silico procedure provided the distribution of estimated digestion sites and of resultant fragment lengths.

\section{Genotyping}

The restriction enzymes MspI $\left(5^{\prime} \mathrm{C} / \mathrm{CGG} 3^{\prime}\right)$ and EcoRI (5'G/AATTC3') were selected for constructing ddRADseq libraries; the modified method described by Peterson et al. [16] was used. After digestion of the genomic DNA at $37{ }^{\circ} \mathrm{C}$, DNA fragments were purified and ligated to MspI and EcoRI adapters. The MspI adapter contains a 4 to 6 nucleotide barcode for sample recognition. DNA fragments between 300 and 500 bp were separated and enriched by PCR amplification. The PCR products were gel-purified and used for pair-end sequencing on the Illumina High-seq2500 sequencing platform (Illumina, Inc.; San Diego, CA, U.S.), following standard protocols.

Based on the Illumina raw data, a custom Perl script was used to sort sequences of individual samples based on indices and trimmed barcode sequences for faster processing. Only sequences containing the barcode followed by an EcoRI or MspI recognition site were retained. Lowquality, contaminant sequences were further filtered using the NGS QC Toolkit [40] and those with more than three missing nucleotides were deleted. The clean data was then mapped to the wild lotus draft genome [1] using the BWA program [18]. Sequences that contained more than two mismatches and multi-mapping reads were excluded. The remaining high quality reads were used for SNP calling using Unified Genotyper in the Genome Analysis Toolkit v3.1-1 (GATK) [19]. The SNPs were filtered as follows: cluster window size 10 , quality of depth (QD) $<3$ and genotype quality $(\mathrm{GQ})<20$. For each given SNP site, the genotypes were labeled as "A" for a homozygous genotype of reference alleles, "B" for homozygous genotype of alternate non-reference alleles, and " $\mathrm{H}$ " for the heterozygous genotype. The low quality (QD <3) and clustered SNP sites were removed from the genotype data, and the genotypes occupying lower reliability $(\mathrm{GQ}<20)$ were replaced by a missing value.

\section{Genetic map construction and anchoring the lotus wild strain genome assembly}

SNP calling was carried out using GATK (v3.1-1) [19] and filtered at the population level according to the following criteria: sequencing depth of SNP sites between 40 and 10,000; mutation rate at each SNP site of less than $5 \%$; and, fewer than $10 \%$ missing SNP sites. SNPs that were heterozygous in both parents were also excluded. The remaining SNP markers were used to construct a linkage map using JoinMap4.1 software [20]. Initial linkage groups (LGs) were established at a LOD threshold of 5 to 20. Using a LOD threshold of 15, most markers could be assigned to 8 LGs. After the markers were grouped, a draft genetic map was constructed. The data points where the genotype data were in disagreement with both flanking data were defined as "singletons." Along the marker order, the markers with more than five "singletons" were excluded using Microsoft Excel, and then the remaining markers were reordered. After grouping, an in-house Perl script (https://github.com/Nextomics/ddRAD-pipeline) was used to trim marker orders by moving the markers in the same scaffold, and to determine marker orientation in linkage group. The SNP markers were further scanned in $100 \mathrm{~kb}$ windows on the mapped scaffolds of the reference genome, and then renamed bins were created to represent continuous co-segregation markers. This process was repeated until deleting markers did not affect marker order. Markers orientation was determined by the trend of linkage distance. Then a new genetic map was constructed and the Kosambi function was used to convert recombination frequencies to relative distances in centimorgans $(\mathrm{cM})$.

After the bin markers had been ordered on the genetic map, the position of the scaffold was then anchored on the basis of bin marker order. Scaffolds containing two or more bin markers were further oriented based on the order of the bin markers on the genetic map and their position on the scaffold.

\section{Linkage map comparison}

The constructed genetic map was compared with maps of $N$. nucifera 'China Antique' and N. lutea 'AL1'. Primers for the SSR markers were first aligned to the reference genome with Bowtie software (v2.2.3) [41], then aligned SSR markers with short repeat sequence domains were used to further anchor the scaffolds of the constructed genetic map and marker positions on the genetic map were confirmed. 


\section{Additional files}

Additional file 1: Distribution of predicted restriction fragments in silico digested with five enzyme combinations. (TIF $298 \mathrm{~kb}$ )

Additional file 2: The basic sequencing data information of the parents and $F_{2}$ progenies. (XLSX $25 \mathrm{~kb}$ )

Additional file 3: Grouped SNPs' name, position on the scaffold and segregation information in the parents and the $\mathrm{F} 2$ individuals. (XLS $13352 \mathrm{~kb}$ )

Additional file 4: The high-density lotus genetic map consisted with 8 LGs (LG1- LG8, on top of the map). The bin names and locations are labeled on the LGs. (PDF $1069 \mathrm{~kb}$ )

Additional file 5: The mapped SNP markers' name, position, type and their flanking sequences and the bin marker information. (XLSX 829 kb)

\section{Abbreviations}

BWA, Burrows-Wheeler Aligner; CM, centimorgan; CTAB, cetyltrimethyl ammonium bromide; ddRADseq, double digest restriction site-associated DNA sequencing GATK, Genome Analysis Toolkit; GQ, genotype quality; LG, linkage group; LOD, "logarithm of the probability ratio", a well-established linkage analysis; MAS, marker-assisted selection; NGS, Next-generation sequencing; PCR, polymerase chain reaction; PVP 40, polyvinylpyrrolidone ( $N$-vinyl pyrrolidone, where $N=40$ ); $\mathrm{QD}$, quality of depth; QTL, quantitative trait locus; RADseq, restriction site-associated DNA sequencing; SGR, segregation distortion region; SNP, Single-nucleotide polymorphism; SSR, Simple Sequence Repeat

\section{Acknowledgements}

We thank associate professor Hongbo Guo (College of Life Science, Northwest A\&F University, Yangling, 712100, P. R. China) and Professor Yuyang Zhang (College of Horticulture \& Forestry, Huazhong Agricultural University) for critical reading the manuscript.

\section{Funding}

This research was funded by (1) National Key Technology R\&D Program (grant no. 2012BAD27B00), (2) Wuhan "Yellow Crane Outstanding Person" program (Wu talents 2013-3).

\section{Availability of data and materials}

The restriction site associated DNA sequencing (RADseq) reads supporting the conclusions of this article have been deposited at NCBI SRA database with accession number PRJNA308152 and hyperlink to datasets in http://www.ncbi.nlm.nih.gov/bioproject/PRJNA308152. Besides, all the SNPs information of the genetic map is provided in additional files.

\section{Authors' contributions}

Conceived and designed the experiments: ZWL, WDK, DPW. Provided the materials: HLZ, YPL. Performed the experiments and identified the plant materials: ZWL, JK, KZ. Analyzed the data: FL, ZHL, DPW. Wrote the paper: ZWL, FL. All authors read and approved the final manuscript.

\section{Competing interests}

The authors declare that they have no competing interests.

\section{Ethics approval and consent to participate}

Not applicable.

\section{Received: 3 February 2016 Accepted: 26 May 2016}

Published online: 17 June 2016

\section{References}

1. Ming R, VanBuren R, Liu Y, Yang M, Han Y, Li LT, Zhang Q, Kim MJ, Schatz MC, Campbell M. Genome of the long-living sacred lotus (Nelumbo nucifera Gaertn). Genome Biol. 2013;14(5):R41.

2. Wang Y, Fan G, Liu Y, Sun F, Shi C, Liu X, Peng J, Chen W, Huang X, Cheng S, et al. The sacred lotus genome provides insights into the evolution of flowering plants. Plant J. 2013;76(4):557-67.

3. Shen-Miller J. Sacred lotus, the long-living fruits of China Antique. Seed Sci Res. 2002;12(03):131-43.
4. Fu J, Xiang QY, Zeng XB, Yang M, Wang Y, Liu YL. Assessment of the Genetic Diversity and Population Structure of Lotus Cultivars Grown in China by Amplified Fragment Length Polymorphism. J Am Soc Hortic Sci. 2011;136(5):339-49.

5. Hu JH, Pan L, Liu HG, Wang SZ, Wu ZH, Ke WD, Ding Y. Comparative analysis of genetic diversity in sacred lotus (Nelumbo nucifera Gaertn.) using AFLP and SSR markers. Mol Biol Rep. 2012;39(4):3637-47.

6. Guo HB. Cultivation of lotus (Nelumbo nucifera Gaertn. ssp. nucifera) and its utilization in China. Genet Resour Crop Evol. 2009:56(3):323-30.

7. Ke WD, Li F, Liu YP, Sun B. Advance on lotus germplasm resources and current breeding. J Changjiang Vegetables. 2003;4:5-9.

8. Liu YM, Wei Y, Li F, Huang XF, Ke WD. Evolvement and utilization of lotus root (Nelumbo nucifera Gaertn.) cultivars in Hubei province. Hubei Agricultural Sciences. 2006;45(3):342-4.

9. Yang M, Han YN, VanBuren $R$, Ming R, Xu LM, Han YP, Liu YL. Genetic linkage maps for Asian and American lotus constructed using novel SSR markers derived from the genome of sequenced cultivar. BMC Genomics. 2012;13(1):653.

10. Zhang Q, Li LT, VanBuren R, Liu YL, Yang M, Xu LM, Bowers JE, Zhong CH, Han YP, Li SH,et al. Optimization of linkage mapping strategy and construction of a high-density American lotus linkage map. BMC Genomics. 2014;15(1):372.

11. Li RQ, Fan W, Tian G, Zhu HM, He L, Cai J, Huang QF, Cai QL, Li B, Bai YQ. The sequence and de novo assembly of the giant panda genome. Nature. 2010;463(7279):311-7.

12. Huang SW, Li RQ, Zhang ZH, Li L, Gu XF, Fan W, Lucas WJ, Wang XW, Xie BY, Ni PX, et al. The genome of the cucumber, Cucumis sativus L. Nat Genet. 2009:41(12):1275-81.

13. Velasco R, Zharkikh A, Affourtit J, Dhingra A, Cestaro A, Kalyanaraman A, Fontana P, Bhatnagar SK, Troggio M, Pruss D, et al. The genome of the domesticated apple (Malus x domestica Borkh.). Nat Genet. 2010;42(10):833-9.

14. Davey JL, Blaxter MW. RADSeq: next-generation population genetics. Brief Funct Genomics. 2010;9(5-6):416-23.

15. Baird NA, Etter PD, Atwood TS, Currey MC, Shiver AL, Lewis ZA, Selker EU, Cresko WA, Johnson EA. Rapid SNP Discovery and Genetic Mapping Using Sequenced RAD Markers. PLoS One. 2008;3(10):e3376.

16. Peterson BK, Weber JN, Kay EH, Fisher HS, Hoekstra HE. Double digest RADseq: an inexpensive method for de novo SNP discovery and genotyping in model and non-model species. PLoS One. 2012;7(5):e37135.

17. Yang M, Han YN, Xu LM, Zhao JR, Liu YL. Comparative analysis of genetic diversity of lotus (Nelumbo) using SSR and SRAP markers. Sci Hortic. 2012;142:185-95.

18. Li H, Durbin R. Fast and accurate short read alignment with BurrowsWheeler transform. Bioinformatics. 2009;25(14):1754-60.

19. McKenna A, Hanna M, Banks E, Sivachenko A, Cibulskis K, Kernytsky A, Garimella K, Altshuler D, Gabriel S, Daly M, et al. The genome analysis toolkit: a MapReduce framework for analyzing next-generation DNA sequencing data. Genome Res. 2010;20(9):1297-303.

20. Van Ooijen JW. Multipoint maximum likelihood mapping in a full-sib family of an outbreeding species. Genet Res (Camb). 2011;93(5):343-9.

21. Singer T, Fan YP, Chang HS, Zhu T, Hazen SP, Briggs SP. A high-resolution map of Arabidopsis recombinant inbred lines by whole-genome exon array hybridization. Plos Genetics. 2006:2(9):1352-61.

22. McCouch SR, Teytelman L, Xu Y, Lobos KB, Clare K, Walton M, et al. Development and mapping of 2240 new SSR markers for rice (Oryza sativa L.). DNA Res. 2002;9(6):199-207

23. Barchi L, Lanteri S, Portis E, Acquadro A, Vale G, Toppino L, Fu B, Maghirang $R$, Li Z, Xing Y, et al. Identification of SNP and SSR markers in eggplant using RAD tag sequencing. BMC Genomics. 2011;12(1):93-8.

24. Guo F, Yu HW, Tang Z, Jiang XL, Wang L, Wang X, et al. Construction of a SNP-based high-density genetic map for pummelo using RAD sequencing. Tree Genetics \& Genomes. 2015:11(1):1-11.

25. Gonen S, Lowe NR, Cezard T, Gharbi K, Bishop SC, Houston RD. Linkage maps of the Atlantic salmon (Salmo salar) genome derived from RAD sequencing. BMC Genomics. 2014;15:166.

26. Celton JM, Christoffels A, Sargent DJ, Xu XM, Rees DJG. Genome-wide SNP identification by high-throughput sequencing and selective mapping allows sequence assembly positioning using a framework genetic linkage map. BMC Biol. 2010;8(1):155.

27. Kai W, Nomura K, Fujiwara A, Nakamura Y Yasuike M, Ojima N, Masaoka T, Ozaki A, Kazeto Y, Gen K, et al. A ddRAD-based genetic map and its 
integration with the genome assembly of Japanese eel (Anguilla japonica) provides insights into genome evolution after the teleost-specific genome duplication. BMC Genomics. 2014;15:233.

28. Kikuchi K, Kai W. Integration of the genetic map and genome assembly of fugu (the tiger pufferfish) and its applications. Nippon Suisan Gakkaishi. 2012;78(2):269.

29. Kundu A, Chakraborty A, Mandal NA, Das D, Karmakar PG, Singh NK, Sarkar D. A restriction-site-associated DNA (RAD) linkage map, comparative genomics and identification of QTL for histological fibre content coincident with those for retted bast fibre yield and its major components in jute (Corchorus olitorius L., Malvaceae s. I.). Mol Breed. 2015;35(1):1-17.

30. Chutimanitsakun Y, Nipper RW, Cuesta-Marcos A, Cistue L, Corey A, Filichkina T, Filichkina T, Johnson EA, Hayes PM. Construction and application for QTL analysis of a Restriction Site Associated DNA (RAD) linkage map in barley. BMC Genomics. 2011;12:4

31. Wu K, Liu HY, Yang MM, Tao Y, Ma HH, Wu WX, Zuo Y, Zhao YZ. Highdensity genetic map construction and QTLs analysis of grain yield-related traits in Sesame (Sesamum indicum L.) based on RAD-Seq techonology. BMC Plant Biol. 2014;14(1):1-14.

32. Li B, Tian L, Zhang JY, Huang L, Han FX, Yan SR, Wang LZ, Zheng HK, Sun JM. Construction of a high-density genetic map based on large-scale markers developed by specific length amplified fragment sequencing (SLAF-seq) and its application to QTL analysis for isoflavone content in Glycine max. BMC Genomics. 2014;15(1):1086.

33. Zhou XJ, Xia YL, Ren XP, Chen YL, Huang L, Huang SM, Liao BS, Lei Y, Yan $L Y$, Jiang HF. Construction of a SNP-based genetic linkage map in cultivated peanut based on large scale marker development using next-generation double-digest restriction-site-associated DNA sequencing (ddRADseq). BMC Genomics. 2014;15(1):351.

34. Diao Y, She CW, Hu ZL. Karyotype Analysis for Zygotene Chromosome of Nelumbo nucifera. Amino Acids \& Biotic Resources. 2006;28(3):34-5.

35. Kianian SF, Quiros CF. Generation of a Brassica oleracea composite RFLP map: linkage arrangements among various populations and evolutionary implications. Theor Appl Genet. 1992;84(5-6):544-54.

36. Zhang Q, Ma B, Li H, Chang Y, Han Y, Li J, Wei G, Zhao S, Khan MA, Zhou Y, et al. Identification, characterization, and utilization of genome-wide simple sequence repeats to identify a QTL for acidity in apple. BMC Genomics. 2012;13:537.

37. Lu H, Romero-Severson J, Bernardo R. Chromosomal regions associated with segregation distortion in maize. Theor Appl Genet. 2002;105(4):622-8.

38. Xu Y, Zhu L, Xiao J, Huang N, McCouch SR. Chromosomal regions associated with segregation distortion of molecular markers in F2, backcross, doubled haploid, and recombinant inbred populations in rice (Oryza sativa L.). Mol Gen Genet. 1997;253(5):535-45.

39. Wang JY, Guo ML, Lin WX. DNA preparation method for lotus (Nelumbo nucifera Gaertn.) containing high polysaccharide. Fujian Science and Technology of Rice and Wheat. 2004;22(4):8-9.

40. Patel RK, Jain M. NGS QC Toolkit: a toolkit for quality control of next generation sequencing data. PLoS One. 2012;7(2):e30619.

41. Langmead B, Salzberg SL. Fast gapped-read alignment with Bowtie 2. Nat Methods. 2012;9(4):357-9.

\section{Submit your next manuscript to BioMed Central and we will help you at every step:}

- We accept pre-submission inquiries

- Our selector tool helps you to find the most relevant journal

- We provide round the clock customer support

- Convenient online submission

- Thorough peer review

- Inclusion in PubMed and all major indexing services

- Maximum visibility for your research

Submit your manuscript at www.biomedcentral.com/submit
Biomed Central 